\title{
Electronic era elusive
}

\section{John C. Angus and Alan T. Collins}

DIAMOND seems to attract superlatives: the atomic density of diamond is the highest of any terrestrial material, and this, coupled with its strong, directional covalent bonding, gives it the highest known elastic modulus (hence its hardness), the highest room temperature thermal conductivity and the highest speed of sound. Less well known has been diamond's potential as a semiconductor. Diamond is structurally and electronically analogous to silicon. It is a wide-band-gap semiconductor and has an unusually high breakdown voltage and low dielectric constant. These properties, coupled with recent advances in the growth of diamond by chemical vapour deposition at low pressure, have led to speculation that diamond might find widespread application in high-speed electronic devices and devices designed to be operated at high temperature. Synthetic diamonds matching the quality and size of their natural counterparts have been a goal of materials scientists for years, and at the biennial diamond conference last month*, it seemed that this ambition has in part been achieved.

Predictions of a brilliant electronic future for diamond have, up to now, been unfulfilled because of the poor quality of synthetic diamond grown from the vapour and because of inherent problems with doping diamond to achieve appropriate conductivities. But researchers at Kobe Steel USA have been growing boron-doped, p-type synthetic diamond films on natural diamond substrates by chemical vapour deposition, and have produced films that have hole mobilities approaching those of the best observed natural diamonds. The mobility of charge carriers is a particularly sensitive indicator of the crystalline perfection of the host lattice (and is essential for effective conduction of charge). Their measurements showed hole mobilities of $1,400 \mathrm{~cm}^{2} \mathrm{~V}^{-1} \mathrm{~s}^{-1}$ at room temperature (J. Glass, Kobe Steel) which can be compared with typical mobilities in natural type II-b semiconducting diamond that range from 500 to $1,000 \mathrm{~cm}^{2} \mathrm{~V}^{-1} \mathrm{~s}^{-1}$ (the best ever reported is $2,000 \mathrm{~cm}^{2} \mathrm{~V}^{-1} \mathrm{~s}^{-1}$ ). In addition, the level of undesired impurities that compensate the charge has been reduced to $5 \times 10^{15} \mathrm{~cm}^{-3}$, equal to that in typical type II-b natural diamond, the standard for diamond quality.

*Fourth International Conference on the New Diamond Science and Technology, Kobe, Japan, 18-22 July 1994.
One long-term objective of research on the electronic properties of diamond is the development of semiconductor devices that can operate at high temperatures, taking advantage of diamond's high band gap and thermal stability. The Kobe research group can now operate diamond field-effect transistors up to $400{ }^{\circ} \mathrm{C}$, and construct simple logic devices.

the growth conditions used for thin-film diamond, carbon prefers to exist as the thermodynamically stable phase, graphite. This makes it all the more difficult to develop synthesis methods and ion implantation processes for doping.

Not all of the reports were so positive. The goal of n-type diamond, important for device development, appears as elusive as ever. In n-type semiconductors current is carried by electrons donated by dissolved impurities that contribute a mobile electron to the crystal. From simple semiconductor theory the group $\mathrm{V}$ elements are expected to form these donor centres in diamond. Nitrogen, which is adjacent to carbon in the periodic table, is readily incorporated into diamond, but the extra electron is too strongly bound to produce significant conductivity. Phosphorus, the next group V element, which is widely used for doping silicon, is too insoluble in diamond to act as an effective dopant. In principle, the solubility limit can be overcome by using ion implantation, but despite sophisticated ion implantation schemes (Johan Prins, Univ. Witwatersrand, South Africa; Rafi Kalish, Technion, Haifa, Israel), stable reproducible n-type doping has not been achieved.

There has been a history of over-optimistic predictions for the application of diamond as an electronic material and, despite the real progress reported at the Kobe

Diamond films of very high quality can also be grown on silicon carbide (Hiroshi Kawarada, Waseda Univ., Tokyo). Although not technically single crystals, these films exhibit a mosaic structure in which the mean angular deviation of the individual crystals is only $2^{\circ}$. The key to these highly oriented films is a rather mysterious pretreatment of the substrate surface with an in situ bombardment obtained by imposing a negative bias of several hundred volts on the surface. This process, first introduced by Shigemi Yugo (Univ. Electro-Communications, Tokyo), has never been satisfactorily explained, but it provides a large number of diamond nuclei which are subsequently subjected to conditions that favour the growth of crystallites with their principal cubic axis perpendicular to the substrate. The result is a mosaic film approaching single-crystal quality. The figure shows an intermediate stage in the development of an oriented diamond film on the cubic face of silicon. The oriented crystallites have just started to grow together.

These achievements are particularly noteworthy because diamond synthesis by chemical vapour deposition is immature compared with silicon and gallium arsenide technologies. In addition, under meeting, there are no active diamondbased electronic devices being marketed (although there is a market for diamond substrates for heat removal from electronic components). Large-scale application of active components will probably require further improvements in quality and decreases in cost.

Future electronic applications may lie in other directions. The use of diamond as an electron emitter was the most promising application discussed at the Kobe meeting. Properly treated diamond surfaces show a negative electron affinity: mobile electrons in the conduction band are at higher energies than electrons at rest in the adjacent vacuum, so they can easily escape from the surface. Possible devices include flat-panel computer and television displays, discharge lamps, cathodes and electron multipliers. If suitable reproducibility, long-term stability and current densities can be achieved, the market for them could be enormous.

John C. Angus is in the Chemical Engineering Department, Case Western Reserve University, Cleveland, Ohio 44106, USA. Alan T. Collins is in the Wheatstone Physics Laboratory, King's College London, Strand, London WC2R 2LS, UK. 